\title{
Narrativa
}

\section{Molte Italie in una città: appunti su presenza e diffusione della cultura italiana a Toronto}

\section{Luca Somigli}

\section{(2) OpenEdition}

\section{Journals}

\section{Edizione digitale}

URL: https://journals.openedition.org/narrativa/868

DOI: $10.4000 /$ narrativa.868

ISSN: 2804-1224

\section{Editore}

Presses universitaires de Paris Nanterre

\section{Edizione cartacea}

Data di pubblicazione: 1 décembre 2016

Paginazione: 153-162

ISBN: 978-2-84016-266-7

ISSN: 1166-3243

\section{Notizia bibliografica digitale}

Luca Somigli, «Molte Italie in una città: appunti su presenza e diffusione della cultura italiana a

Toronto», Narrativa [Online], 38 | 2016, online dal 01 janvier 2022, consultato il 22 février 2022. URL:

http://journals.openedition.org/narrativa/868 ; DOl: https://doi.org/10.4000/narrativa.868

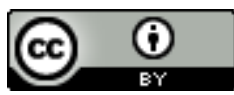

Narrativa est mise à disposition selon les termes de la Licence Creative Commons Attribution 4.0 International. 


\section{Molte Italie in una città: appunti su presenza e diffusione della cultura italiana a Toronto}

P arlare di diffusione e ricezione della letteratura italiana fuori d'Italia vuol dire parlare, a mio parere, di almeno due fenomeni molto diversi: da una parte, la diffusione di opere in traduzione, rivolte potenzialmente all'intero pubblico di lettori nella lingua d'arrivo; dall'altra, quella di testi in italiano, rivolti invece a un pubblico relativamente ristretto, spesso composto per lo più da immigrati antichi o recenti, con diverse competenze linguistiche e diversi livelli di scolarizzazione. Questa premessa mi serve per spiegare la ragione per cui il mio intervento si focalizza su un contesto geografico e culturale molto preciso, la città di Toronto, piuttosto che più genericamente sul Canada. Sarebbe infatti molto difficile poter identificare delle tendenze specificamente canadesi riguardo al primo tipo di diffusione della letteratura italiana, quello cioè dei testi in traduzione. Se esiste in Canada un'attenta politica di difesa della produzione culturale nazionale nelle due lingue ufficiali, l'inglese e il francese, grazie anche ad agenzie governative provinciali e federali a ciò preposte, quando si tratta di importare prodotti culturali stranieri, il Canada non è che una provincia relativamente piccola del mondo anglofono o della Francophonie. In altre parole, la diffusione di testi stranieri (letterari o meno) risponde nella maggior parte dei casi alle strategie e alle scelte editoriali delle grandi case editrici che fanno capo a New York, Londra o Parigi.

È invece a livello locale che possiamo trovare numerosi attori pubblici e privati impegnati nella promozione della cultura italiana, e allo stesso tempo un pubblico relativamente ampio e stratificato la cui fruizione di prodotti culturali italiani spazia dall'ambito accademico e scientifico all'intrattenimento e all'informazione. Non bisogna infatti dimenticare che Toronto ospita una delle più grandi comunità di origine italiana del mondo. Nel censimento realizzato dal governo canadese nel 2011, circa 475.000 residenti nella zona metropolitana di Toronto si sono autodefiniti di origine italiana. Di questi, quasi 167.000 hanno 
indicato l'italiano come lingua-madre, e 65.000 anche come lingua parlata in casa ${ }^{1}$. La durevolezza e la relativa vitalità dell'italiano a Toronto e nell'Ontario, la provincia di cui è capitale, si spiegano con il fatto che l'immigrazione italiana in Canada ha avuto il suo picco nel secondo dopoguerra, piuttosto che nel periodo fra le due guerre come nel caso degli Stati Uniti, e si registrano numeri elevati di nuovi arrivi fino a tutti gli anni ' $80^{2}$. Gli immigrati delle ultime generazioni tendevano a essere più scolarizzati rispetto ai loro predecessori, meglio integrati nella comunità nazionale da cui erano partiti, e meglio capaci di mantenere con l'Italia un rapporto continuativo grazie ai mass media e a nuovi mezzi di comunicazione da un lato e alla maggiore facilità di tornare in Italia anche per brevi periodi, resa possibile dall'espansione e dalla relativa accessibilità dei voli intercontinentali dall'altro. Inoltre, per quanto disseminata in tutto il paese, l'immigrazione italiana ha teso a concentrarsi in Québec e in Ontario, e in particolare nei due maggiori centri urbani, Toronto e Montréal. Qui, già nel periodo tra le due guerre si è avuta la formazione di "due consistenti 'Little Italy', culturalmente e geograficamente ancora riconoscibili e con a disposizione, da diversi anni ormai, degli organi di informazione" - e potremmo aggiungere, di intrattenimento e di diffusione culturale - "in lingua italiana"3. A partire dal secondo dopoguerra, la comunità italofona di Toronto è stata servita da media di ogni sorta: giornali e periodici, in particolare il Corriere canadese, fondato nel 1954 e ancora in vita, per quanto precariamente; stazioni radio, come la ben nota CHIN dell'imprenditore Johnny Lombardi, che fa musica e informazione in italiano dal 1967; e televisione, con la sezione italiana di OMNI Television, che propone programmi sia prodotti localmente che acquistati dalla RAI (fra i più recenti, anche Montalbano). Tra gli anni '60 e gli anni '80, a Toronto si potevano facilmente vedere film in italiano grazie a sale cinematografiche

1. I dati del censimento si possono trovare sul sito di "Statistics Canada", http:// www12.statcan.gc.ca/census-recensement/index-eng.cfm. Per un'analisi dei dati linguistici, si veda anche PIERno, Franco, "Gli italianismi nell'inglese di Toronto e nel francese di Montréal. Stato delle ricerche e possibilità di collaborazione con l'OIM", Atti della giornata di studio dell'OIM (Osservatorio degli italianismi nel Mondo), 24 gingno 2014, a cura di Luca Serianni e Matthias Heinz, Firenze, Accademia della Crusca, in corso di stampa.

2. Per un quadro generale sull'immigrazione italiana in Canada, si veda De MAriA Harney, Nicholas, Eh, Paesan! Being Italian in Toronto, Toronto, University of Toronto Press, 1998, che comprende anche statistiche comparative dei flussi migratori dall'Italia verso il Canada e gli USA.

3. Ibid. 
specializzate e all'attività di club e associazioni di immigrati ${ }^{4}$, così come si potevano acquistare libri in due librerie italiane, ancora aperte - ma per poco quando chi scrive è arrivato in Canada nel 1997. Vale la pena di sottolineare che il materiale reso disponibile da queste iniziative commerciali non era, per la gran parte, il cinema d'autore distribuito nei circuiti d'essai in tutto il Nord America o la letteratura alta, ma il cinema di consumo, dalla commedia all'italiana al poliziottesco a, addirittura, film americani doppiati, o la narrativa gialla o rosa, spesso tradotta dall'inglese - insomma, tutta quella produzione culturale media e medio-bassa per un pubblico generico che evidentemente era abbastanza vasto da sostenerne, per quanto precariamente e temporaneamente, il mercato.

Della presenza di una forte comunità italiana ha beneficiato a lungo il sistema scolastico, dalla scuola dell'obbligo all'università, non solo della città di Toronto ma dell'intera provincia dell'Ontario. Maddalena Kuitunen e Julius Molinaro così descrivono gli immigrati del periodo post-bellico: "Accettati in genere dalla nuova politica immigratoria canadese, assistiti nell'espatrio anche dal governo italiano $[. .$.$] , socialmente più evoluti, i nuovi italo-canadesi vedevano in numero$ sempre maggiore l'università come lo sbocco naturale per i loro figli’" ${ }^{5}$. Se, una volta giunti all'università, molti di questi giovani frequentavano poi i corsi di lingua e letteratura italiana non era soltanto per una questione d'identità culturale, ma anche perché una laurea in quella disciplina risultava spendibile nel mondo del lavoro, in particolare nelle scuole in cui si moltiplicarono le cattedre di lingua italiana. In una sorta di circolo virtuoso, purtroppo spezzatosi da più di un decennio, la richiesta di insegnanti di lingua nella scuola dell'obbligo si traduceva a sua volta in corsi di laurea nelle maggiori università dell'intera provincia, e non solo delle due università della capitale, la York University e l'Università di Toronto.

Se a questo punto mi soffermerò in particolare sul secondo ateneo non è per motivi di bandiera, ma perché il Dipartimento di Studi Italiani dell’Università di Toronto in effetti rappresenta il centro principale dell'italianistica canadese (è il

4. L'imprenditore di origini pugliesi Rocco Mastrangelo, proprietario tra gli anni '60 e gli anni '80 di una catena di cinema italiani a Toronto, ha recentemente donato all'Università di Toronto una collezione di ben 1800 film in $35 \mathrm{~mm}$. all'epoca programmati nei suoi locali, che spaziano un po' in tutta la produzione italiana della seconda metà del Novecento. Una breve intervista con Mastrangelo disponibile su Youtube (https://www.youtube.com/watch?v=8FL9pkFBbwA) fornisce un'interessante testimonianza sulla presenza della cultura popolare italiana a Toronto.

5. Kuitunen, Maddalena, Molinaro, Julius A., A History of Italian Studies at the University of Toronto (1840-1940). Lo studio dell'italiano all'Università di Toronto, Toronto, Department of Italian Studies, University of Toronto, 1991, p. 147. 
solo nel paese a conferire l'intera gamma di titoli accademici in studi italiani, dal BA, equivalente grossomodo alla laurea triennale italiana, al dottorato di ricerca, o $\mathrm{PhD}$ ), e uno dei maggiori in Nord America ${ }^{6}$. Per quanto l'italianistica torontoniana possa vantare un pedigree che risale addirittura a prima della fondazione del Canada e dell'unificazione italiana - il primo docente di italiano fu il patriota ed esule James Forneri, giunto a Toronto nel 1853 - è solo negli anni '50, con la formazione dei primi $\mathrm{PhD}$, che il dipartimento di italiano, unito fino al 1973 a quello di spagnolo, inizia a emergere come un importante centro di ricerca ${ }^{7}$. L'attività scientifica era inizialmente orientata sul Rinascimento, ambito in cui si è venuta a creare una vera e propria scuola, ma dalla metà degli anni ' 70 si può notare un sensibile e repentino aumento delle tesi di argomento novecentesco, con, in anni recenti, una grande attenzione per la contemporaneità, grazie anche al lavoro di Rocco Capozzi, che ha diretto tesi su, fra gli altri argomenti, Calvino (Franco Ricci, 1983; Franco Gallippi, 2007), la narrativa sperimentale degli anni '60 (Paolo Chirumbolo, 2004), Celati (Anna Maria Chierici, 2010), Malerba (Anna Chiafele, 2010) e Tondelli (Sciltian Gastaldi, 2012). L'attenzione costante per la contemporaneistica è testimoniata anche dall'attività di ricerca del dipartimento. Convegni come Scrittori, tendenze letterarie e conflitto delle poetiche in Italia (1960-1990), organizzato da Rocco Capozzi e Massimo Ciavolella nel 1990 e cui parteciparono fra gli altri Romano Luperini, Renato Barilli, Sandro Briosi, Biancamaria Frabotta, Silvio Ramat e Lamberto Pignotti, o come, se mi si permette un momento di autoreferenzialità, Negli archivi e per le strade. Il ritorno alla realtà nella narrativa di inizio millennio, organizzato dal sottoscritto nel 2010, intendevano fornire un luogo in cui studiosi di diversa formazione e nazionalità potessero incontrarsi per cercare di fare il punto su alcune delle tendenze - come recitava appunto il titolo del convegno del 1990 - del discorso letterario e critico in Italia, e si proponevano quindi come spazio di mediazione della cultura letteraria italiana in Nord America.

Qualche parola merita poi l'importante opera di diffusione della cultura italiana svolta dalla University of Toronto Press, una delle maggiori case editrici accademiche in Nord America e la più prestigiosa del Canada. La University of Toronto Press ha al suo attivo ben due collane dedicate alla letteratura e cultura italiana. La prima e più antica, la "Toronto Italian Studies", è soprattutto il

6. In Canada, solo la McGill University, università anglofona di Montréal, ha un programma di dottorato ad hoc; vale la pena di notare che non esistono cattedre universitarie d'italiano nelle università francofone del Québec.

7. Prima degli anni '50, era stato conferito un solo dottorato in italiano, nel 1932, a Beatrice Corrigan, poi a lungo docente all’Università di Toronto. 
frutto dell'intelligenza, acume e passione di una straordinaria figura di editor, Ron Schoeffel (1936-2013), "a gentleman and a scholar", come lo definì giustamente il quotidiano Globe and Mail nell'annunciarne la scomparsa. ${ }^{8}$. Una semplice scorsa dei titoli pubblicati dalla metà degli anni ' 90 ad oggi, il periodo per il quale è disponibile una lista completa ${ }^{9}$, mostra un costante aumento del ritmo delle pubblicazioni, con picchi di 17 volumi nel 2015, e 15 nel 2011 e nel 2014. L'investimento negli studi italiani all'inizio del nuovo secolo, quando la politica editoriale delle altre grandi university presses nordamericane andava nella direzione opposta, ha permesso alla casa editrice di attrarre collaboratori non più solo dal Canada, come avveniva ancora negli anni ’90, ma anche dagli Stati Uniti e dagli altri paesi anglofoni. In questo modo, la collana "Toronto Italian Studies" è divenuta il maggior punto di riferimento per gli studi italiani in Nord America, e il suo catalogo attuale fornisce molti elementi utili per comprendere gli orientamenti dell'italianistica nell'intero contesto continentale, in cui riesce sempre meno facile (e utile) distinguere tra Canada e Stati Uniti. L'immagine che le pubblicazioni più recenti offrono è quella di una disciplina con un profilo sempre più specifico e differenziato rispetto all'italianistica italiana, con una forte apertura al dialogo con le più recenti tendenze critiche e teoriche degli studi culturali nordamericani: penso ad esempio a lavori come The Beautiful Country: Tourism and the Impossible State of Destination Italy (2015), di Stephanie Malia Hom, sull'evoluzione del concetto di "Italia" come destinazione turistica, che si colloca all'intersezione tra critica culturale, geografia e "tourism studies", o come la raccolta di saggi Stillness in Motion: Italy, Photography, and the Meanings of Modernity, a cura di Sarah Patricia Hill e Giuliana Minghelli, che affronta da una varietà di prospettive critiche l'evolversi del ruolo e dello statuto della fotografia all'interno del sistema culturale italiano.

Alla diffusione della letteratura italiana in traduzione sta dando invece un importante contributo l'altra iniziativa editoriale della University of Toronto Press, che vede anche il patrocinio del Ministero degli Affari Esteri e del Ministero per i Beni e le Attività Culturali. Si tratta della "Lorenzo da Ponte Library", una "collezione organica di testi italiani in traduzione inglese", come recita il sito ad essa dedicato, il cui scopo è "rendere disponibili cento opere di autori italiani che costituiscono significativi contributi letterari, filosofici, giuridici e

8. KirchHof, Jack, “Superman Editor' Ron Schoeffel Lived for Scholarship”, The Globe and Mail, 1 agosto 2013, http://www.theglobeandmail.com/arts/ books-and-media/superman-editor-ron-schoeffel-lived-for-scholarship/article13571385/.

9. Http:/ / www.utppublishing.com/search.php?mode $=$ search\&sby_series $=$ Toronto $\% 20$ Italian $\% 20$ Studies. 
storici al mondo della cultura internazionale"10. La dedica al librettista di Mozart e Salieri non è, come è ovvio, casuale: emigrato negli Stati Uniti nel 1805 per sfuggire ai creditori inglesi, Da Ponte divenne nel 1825 il primo docente di letteratura italiana alla Columbia University, e rappresenta in un certo senso l'inizio di quella tradizione di "Italian Studies" in Nord America che la collana intende autorevolmente interpretare. Diretta da Luigi Ballerini e Massimo Ciavolella, entrambi professori di letteratura italiana all'Università della CaliforniaLos Angeles (ma Ciavolella è stato fino al 1997 docente e per un periodo direttore del Dipartimento di Studi Italiani dell'Università di Toronto), la collana ha ad oggi al suo attivo ventotto dei previsti cento volumi. Nonostante l'evidente intento canonizzante del progetto, la scelta di autori e opere segue logiche più complesse che non una semplice riproposizione dei classici. Testi come il manuale La scienza in cucina (Science in the Kitchen and the Art of Eating Well) di Pellegrino Artusi, che ha inaugurato la collana nel 2003, il Saggio storico sulla rivoluzione napoletana del 1799 (Historical Essay on the Neapolitan Revolution of 1799, 2014) di Vincenzo Cuoco, o il trattato cinquecentesco La vita sobria (Writings on the Sober Life) di Alvise Cornaro sembrano delineare una sorta di contro-canone parallelo a quello dei grandi scrittori nei generi letterari tradizionali che mette in luce il contributo ininterrotto della cultura italiana ai saperi più diversi, dall'arte culinaria alla storiografia a una sorta di foucaltiana "estetica dell'esistenza" avant la lettre. Numerosi volumi miscellanei, come An Italian Renaissance Sextet (2004), From Kant to Croce: Modern Philosophy in Italy, 1800-1950 (2012), o Those Who from Afar Look Like Flies, un'antologia di poesia da Pasolini ai giorni nostri, il cui primo tomo è di prossima uscita, permettono poi di ampliare il raggio della collana oltre i cento autori imposti dal numero di volumi previsto per includere così figure minori e anche minime che contribuiscono ulteriormente ad arricchire il panorama della cultura italiana dalle origini al presente.

Nel disegno originale di questa relazione, all'università come luogo di canonizzazione e storicizzazione della produzione culturale avrebbe dovuto corrispondere l'Istituto Italiano di Cultura di Toronto come luogo di diffusione e, in parte, di sperimentazione. Nonostante il fatto che nell'approfondire quella che potremmo chiamare la "situazione sul campo" mi sia presto reso conto che questa è ben più complessa, lo schema dicotomico che l'opposizione delineava rimane almeno in parte valido. Me lo confermava recentemente l'attuale direttore, Alessandro Ruggera, descrivendo l'attività dell'Istituto come "cultural diplomacy", come promozione della cultura italiana contemporanea in un pro-

10. Http://www.dapontelibrary.com/about.html 
ficuo dialogo e collaborazione con le istituzioni locali, e in alternativa al lavoro di ricerca delle università dell'Ontario, con le quali comunque l'Istituto mantiene un costante rapporto di cooperazione, soprattutto nella sponsorizzazione e gestione di grandi eventi come convegni ${ }^{11}$. Fondato nel 1976 come sezione culturale del Consolato Generale di Toronto, l'Istituto di Toronto ha sede fin dal 1982 su Huron Street, a pochi passi dall'università, in uno spazio polivalente adatto per conferenze, letture, mostre, proiezioni e, in aule apposite, l'insegnamento della lingua ${ }^{12}$. Entro i limiti delle linee-guida generali indicate dal Ministero degli Esteri, da cui gli Istituti dipendono, ai direttori degli istituti è lasciata ampia libertà di movimento per quanto riguarda le modalità con cui condurre questa operazione di “diplomazia culturale". In un’intervista, Carlo Coen, direttore dell'Istituto dall'agosto 1999 all'ottobre 2005, mi ha raccontato ad esempio di essersi reso conto al suo arrivo che vi era un ampio spazio tra la cultura "alta" promossa dall'università, dai musei, dalle sale da concerto o dal teatro dell'opera cittadini, e le fiorenti attività folkloristiche legate alla realtà locale di club e associazioni di immigrati, e che in questo spazio si poteva inserire utilmente l'Istituto $^{13}$. Un esempio fra i molti possibili: Toronto è una delle capitali del cinema internazionale, sia dal punto di vista della produzione (la "Hollywood del Nord", come è spesso chiamata, è il terzo centro di produzione del Nord America dopo New York e Los Angeles) che della promozione, in particolare grazie al Toronto Film Festival, uno dei più importanti festival cinematografici del mondo. Coen ha promosso numerose iniziative legate al cinema italiano, fra queste la partecipazione al progetto "Italia Cinema" e alla fondazione del Toronto Italian Film Festival, nato nel 1999 con lo scopo di portare in Canada film altrimenti non distribuiti in Nord America e allo stesso tempo di valorizzare la produzione di cinema "italo-canadese", cioè legato alle esperienze degli italiani in Canada e/o diretto da registi di origine italiana. Quasi venti anni dopo, l'Istituto rimane uno dei principali sponsor di quello che è in qualche modo il "discendente" di quel progetto, l'Italian Contemporary Film Festival che si tiene nel mese di giugno in varie città dell'Ontario e del Québec.

Ruggera aggiunge che oltre alla collaborazione con le istituzioni culturali canadesi, è necessaria una continuità di interventi, una "serialità", per così dire, che permetta di sviluppare in profondità certi temi e problematiche. Insieme

11. Intervista registrata il 9 maggio 2016.

12. Italian Cultural Institute. Toronto, 1985-91, Toronto, Istituto Italiano di Cultura, senza data (ma 1991), pp. 13-14.

13. Intervista registrata il 25 maggio 2016. 
alla facoltà di architettura dell'Università di Toronto, l'Istituto ha ad esempio in programma una serie di mostre di cui la più recente, intitolata "Public Ambitions", aveva come tema il modo in cui dieci architetti italiani hanno reinterpretato la progettazione di spazi urbani pubblici ${ }^{14}$. Dal 2015, l'Istituto collabora con il Toronto Comics Arts Festival invitando disegnatori e autori di fumetti italiani del calibro di Lorenzo Mattotti e Manuele Fior a esporre le loro tavole negli spazi dell'Istituto. È semmai proprio la letteratura a risultare di più difficile promozione rispetto ad altre forme di produzione culturale, per gli ovvi problemi causati dalla barriera linguistica nel caso degli autori contemporanei spesso poco o per niente tradotti nelle due lingue ufficiali del Canada. Anche in questo caso, vi sono dei partner locali con cui collaborare, in particolare il prestigioso International Festival of Authors, una vera e propria istituzione canadese fin dalla sua fondazione nel 1974, che ha avuto tra i suoi ospiti Umberto Eco, Claudio Magris e Sandro Veronesi. È su questo terreno che si possono poi ancora incontrare gli interessi e il mandato dell'Istituto e delle varie istituzioni preposte allo studio della letteratura, come nel recente caso della sponsorizzazione da parte del primo della lezione magistrale dello scrittore Amara Lakhous in occasione del congresso della Canadian Society for Italian Studies del 2016.

La diffusione della letteratura, però, deve passare per altri canali. Si è detto sopra che l'editoria canadese si trova ad essere spesso schiacciata tra i grandi colossi anglo-americani e francesi. Ciò non vuol dire però che non esista anche un'editoria di piccole e medie dimensioni che può farsi promotrice di iniziative originali, spesso legate ai gusti e agli interessi specifici di proprietari, editors o direttori di collana. Si tratta, come è ovvio, di un'editoria di nicchia, rivolta a un pubblico relativamente ristretto, che se da una parte può svolgere un importante ruolo di mediazione culturale ad alto livello, dall'altra è spesso tagliata fuori dalla grande distribuzione e sopravvive grazie a finanziamenti pubblici e sponsorizzazioni semi-private. È questo ad esempio il caso di Guernica Editions, fondata nel 1978 a Montréal dallo scrittore e imprenditore culturale di origini italiane Antonio D'Alfonso (che nel 1994 la trasferì a Toronto) con lo scopo di proporre al pubblico l'opera di poeti che non rientrassero nelle tendenze dominanti della letteratura soprattutto anglofona del periodo. Negli anni, l'offerta di Guernica si è ampliata fino a includere opere di tutti i generi letterari, di memorialistica e di saggistica, mentre, come ha rilevato Linda Morra in un profilo della casa editrice, il programma editoriale, influenzato anche dall'ascesa del multiculturalismo come paradigma sociale e culturale del paese, si è indiriz-

14. https://www.daniels.utoronto.ca/events/event/2016-04-07/public-ambitions 
zato verso la valorizzazione della produzione letteraria di gruppi ai margini del mainstream canadese ${ }^{15}$. Non è comunque mai venuta meno l'attenzione per la letteratura e soprattutto per la poesia italiana, uno dei principali interessi di D'Alfonso. La lista di poeti apparsi in traduzione inglese presso Guernica è un vero e proprio $W$ ho's $W$ ho della poesia del dopoguerra, e spazia da Mario Luzi a Vittorio Sereni, da Giorgio Caproni a Luciano Erba, da Antonio Porta a Maria Luisa Spaziani, da Biancamaria Frabotta a Edoardo Sanguineti, senza trascurare la Svizzera italiana, rappresentata da Giorgio Orelli e Fabiano Alborghetti, e la poesia di inizio millennio, con due vincitori del Premio Montale come Antonella Anedda e Tiziano Broggiato. Meno organico il catalogo di opere italiane in prosa o teatrali, che comprende fra le altre Mistero napoletano di Ermanno Rea (A Mistery in Naples, 2004) e Natale in Casa Cupiello di Eduardo De Filippo (The Nativity Scene, 1997). È evidente comunque in queste ultime scelte una certa attenzione a colmare appariscenti lacune nella letteratura contemporanea italiana disponibile in traduzione. In questa direzione si muove anche l'utile iniziativa di due docenti di Toronto, Giuliana Sanguineti Katz e Anne Urbancic, che hanno tradotto insieme opere di Angela Bianchini (The Girl in Black, 2002), Maria Rosa Cutrufelli (Reasonable Doubt, 2007), ed Enzo Lauretta (The Narrow Beach, 2004), pubblicate però da piccoli o minimi editori e con diffusione limitata.

Per concludere poi, alcune riflessioni sulla produzione culturale italiana e italo-canadese a Toronto. Tralascio, per questioni di spazio, l'annoso e complesso problema di cosa si possa intendere con categorie notoriamente fluide come "italo-canadese". Piuttosto, ciò che mi preme sottolineare è che in Canada, e in particolare a Toronto e a Montréal, fiorisce ancora una produzione letteraria in italiano, con una decisa predominanza della poesia ${ }^{16}$. È questo un altro aspetto per il quale la comunità italiana di Toronto differisce da quelle delle principali città statunitensi, ed è ancora una volta il risultato dell'incrocio tra, da un lato, un'immigrazione più recente e, dall'altro, la politica multiculturale del governo canadese, che ha permesso di coltivare la lingua italiana a immigrati di prima e seconda generazione anche al di fuori dell'ambito domestico. La comunità degli scrittori italiani a Toronto include quindi autori formatisi intellettual-

15. Morra, Linda, "Guernica Editions", Historical Perspectives on Canadian Publishing, http:/ /hpcanpub.mcmaster.ca/case-study/guernica-editions.

16. Per quanto riguarda la letteratura in inglese, la comunità italiana di Toronto ha già prodotto autori di rilievo, tra cui Nino Ricci, vincitore di due Governor General's Awards per la narrativa, e Pier Giorgio Di Cicco, poeta laureato di Toronto dal 2004 al 2009. 
mente in Italia e che hanno continuato a scrivere in italiano una volta emigrati, come Maria Ardizzi, originaria della provincia di Teramo e nota soprattutto per una trilogia di romanzi dei quali il primo, Made in Italy (1982), ha vinto un premio dell'Ontario Arts Council nel 1980, o Corrado Paina, milanese giunto trentenne a Toronto dove all'attività di scrittore affianca quella di pubblicista e imprenditore culturale, ma include anche autori nati in Italia e cresciuti in Canada che spesso scrivono sia in inglese che in italiano, come Gianna Patriarca, o addirittura in tutte e tre le lingue nazionali, come il già menzionato Antonio D'Alfonso, perfettamente a suo agio in italiano, inglese e francese (alle quali in alcuni componimenti aggiunge lo spagnolo).

La cultura italiana a Toronto rappresenta infine un punto d'incontro per le varie istituzioni che abbiamo considerato in queste pagine: università, Istituto di Cultura, editoria. Il Dipartimento di Studi Italiani dell'Università di Toronto ospita nelle sue strutture il Centro di Studi Italo-Canadesi Frank Iacobucci e la rivista ad esso associata, Italian-Canadiana. Inoltre, vi è una tradizione ormai radicata di studi linguistici dedicati all'italiano di Toronto, dai primi lavori sul cosiddetto "italiese" al più recente progetto di ricerca, diretto da Franco Pierno, che ha come scopo il censimento e lo studio degli italianismi in Canada, e che fa parte dell'Osservatorio degli Italianismi nel Mondo costituito dall'Accademia della Crusca. L'Istituto ha patrocinato numerose iniziative legate alla letteratura italo-canadese di Toronto, dalla serie di letture di poesia organizzata da chi scrive insieme a Corrado Paina tra l'ottobre 2001 e il marzo 2002 alle presentazioni di libri di autori italiani a Toronto. La piccola e media editoria ha contribuito alla diffusione di questo materiale, sia in maniera programmatica come nel caso già ricordato di Guernica sia come parte di un più ampio catalogo di poesia canadese tout court, come nel caso di Mansfield Press o di Quattro Books.

Parlare di cultura italiana a Toronto significa dunque interrogarsi non solo sui circuiti di diffusione e di ricezione di una cultura prodotta altrove e mediata attraverso istituzioni locali, ma anche sul significato stesso della categoria di "italiano", per la quale i confini nazionali risultano ormai senz'altro stretti.

Luca SomigLi

University of Toronto 